\title{
CD47 Promotes Neuronal Development through Src- and FRG/Vav2-Mediated Activation of Rac and Cdc42
}

\author{
Takaaki Murata, ${ }^{1,2}$ Hiroshi Ohnishi, ${ }^{1}$ Hideki Okazawa, ${ }^{1}$ Yoji Murata, ${ }^{1}$ Shinya Kusakari, ${ }^{1}$ Yuriko Hayashi, ${ }^{1}$ \\ Motoaki Miyashita, ${ }^{1}$ Hiroshi Itoh, ${ }^{3}$ Per-Arne Oldenborg, ${ }^{4}$ Nobuhiko Furuya, ${ }^{2}$ and Takashi Matozaki ${ }^{1}$ \\ ${ }^{1}$ Laboratory of Biosignal Sciences, Institute for Molecular and Cellular Regulation, Gunma University, Maebashi, Gunma 371-8512, Japan, ${ }^{2}$ Department of \\ Otolaryngology, Gunma University Graduate School of Medicine, Gunma 371-8511, Japan, ${ }^{3}$ Department of Cell Biology, Graduate School of Biological \\ Sciences, Nara Institute of Science and Technology, Ikoma, Nara 630-0192, Japan, and ${ }^{4}$ Department of Integrative Medical Biology, Section for Histology \\ and Cell Biology, Umeå University, S-901 87 Umeå, Sweden
}

The development of axons and dendrites is controlled by small GTP-binding proteins of the Rho family, but the upstream signaling mechanisms responsible for such regulation remain unclear. We have now investigated the role of the transmembrane protein cluster of differentiation 47 (CD47) in this process with hippocampal neurons. CD47-deficient neurons manifested markedly impaired development of dendrites and axons, whereas overexpression of CD47 promoted such development. Interaction of SH2 domain-containing protein tyrosine phosphatase substrate-1 (SHPS-1) with CD47 also induced the formation of dendritic filopodia and spines. These effects of CD47 were prevented by inhibition of either cell division cycle 42 (Cdc42) or Rac. In CD47-deficient neurons, autophosphorylation of Src was markedly reduced. In addition, overexpression of CD47 promoted the autophosphorylation of Src. Inhibition of Src family kinases indeed prevented CD47-promoted dendritic development. Inhibition of either FGD1-related Cdc42-guanine nucleotide exchange factor (GEF) (FRG) or Vav2, which is a GEF for Cdc42 and Rac and is activated by Src, also prevented the effects of CD47 on dendritic development. These results indicate that CD47 promotes development of dendrites and axons in hippocampal neurons in a manner dependent, at least in part, on activation of Cdc42 and Rac mediated by Src as well as by FRG and Vav2.

Key words: dendrite; Src; small GTP-binding protein; guanine nucleotide exchange factor; SHPS-1; hippocampus

\section{Introduction}

Regulation of the extension and branching of axons and dendrites is essential for the formation of functional neuronal networks in the mammalian CNS. Such regulation is achieved by a variety of extracellular signaling molecules, including diffusible factors, extracellular matrix proteins, and cell adhesion molecules (Tessier-Lavigne and Goodman, 1996; Dickson, 2002; Jan and Jan, 2003). In response to such extracellular signals, axons or dendrites produce or retract filopodia or lamellipodia, actions that require the temporal and spatial regulation of the actin cytoskeleton. Members of the Rho family of small GTP-binding proteins, including Rho, Rac, and cell division cycle 42 (Cdc42), are implicated as key mediators that link extracellular signals to rearrangement of the actin cytoskeleton in neurons (Luo et al., 1997; Takai et al., 2001; Govek et al., 2005). Indeed, certain neu-

\footnotetext{
Received June 6, 2006; accepted 0ct. 13, 2006.

This work was supported by a Grant-in-Aid for Scientific Research on Priority Areas Cancer, a Grant-in-Aid for Scientific Research on Priority Areas Molecular Brain Science, a grant of the 21st Century COE Program from the Ministry of Education, Culture, Sports, Science, and Technology of Japan, Swedish Research Council Grant 31X14286, National Institutes of Health Grant GM57573-06, and grants from the Swedish Society of Medicine and the Faculty of Medicine, Umeå University, Sweden. We thank Y. Takai, M. Okada, M. Matsuda, and J. Miyazaki for reagents, as well as H. Kobayashi, K. Tomizawa, and Y. Niwayama for technical assistance.

Correspondence should be addressed to Takashi Matozaki, Laboratory of Biosignal Sciences, Institute for Molecular and Cellular Regulation, Gunma University, 3-39-15 Showa-Machi, Maebashi, Gunma 371-8512, Japan. E-mail: matozaki@showa.gunma-u.ac.jp.

DOI:10.1523/JNEUROSCI.3981-06.2006

Copyright $\odot 2006$ Society for Neuroscience $\quad$ 0270-6474/06/2612397-11\$15.00/0
}

ronal guidance factors, including slit, semaphorin, and ephrin, have been shown to regulate Rho family proteins (Polleux et al., 2000; Wahl et al., 2000; Shamah et al., 2001; Whitford et al., 2002). However, the mechanisms responsible for integration of other extracellular signals, such as those generated by adhesion molecules, with Rho family proteins in the promotion of axonal and dendritic development remain poorly understood.

Cluster of differentiation 47 is a member of the Ig superfamily of proteins, possessing an IgV-like extracellular region, five putative transmembrane domains, and a short cytoplasmic tail (Brown and Frazier, 2001). CD47 is expressed throughout the brain (Reinhold et al., 1995), being especially abundant in synapse-rich regions such as the molecular layer and synaptic glomeruli of the cerebellum, the plexiform layers of the retina, and the hippocampus (Jiang et al., 1999; Mi et al., 2000; Ohnishi et al., 2005), and its expression increases markedly during postnatal development (Mi et al., 2000; Ohnishi et al., 2005). In addition, the abundance of CD47 mRNA in the hippocampus correlates with memory retention in rats (Huang et al., 1998), and long-term potentiation is impaired in CD47-deficient mice (Chang et al., 1999), suggesting that CD47 plays a role in synaptic plasticity and memory formation in the hippocampus. However, the precise function of $\mathrm{CD} 47$ in neurons and its mode of action at the molecular level remain essentially unknown.

We have shown recently that forced expression of CD47 promotes neurite formation in N1E-115 neuroblastoma cells in a 
manner dependent on the activation of Rac and integrin $\beta 3(\mathrm{Mi}-$ yashita et al., 2004). Indeed, the extracellular region of CD47 associates with integrin $\beta 3$ or $\beta 1$ subunits, and most CD47mediated responses in non-neuronal cells, such as migration of neutrophils and activation of platelets, appear to require integrin activation (Brown and Frazier, 2001). Moreover, we also shown that forced expression of CD47 or exposure to a recombinant fusion protein of SHPS-1 (SH2 domain-containing protein tyrosine phosphatase substrate-1) and the Fc domain of Ig markedly promoted Cdc42-mediated filopodium formation in N1E115 neuroblastoma cells (Miyashita et al., 2004). SHPS-1, also known as BIT (brain Ig-like molecule with tyrosine-based activation motifs) or $\operatorname{SIRP} \alpha$ (signal-regulatory protein $\alpha$ ), is a ligand for the extracellular region of CD47 (Jiang et al., 1999). It is a transmembrane protein that contains three Ig-like domains in its extracellular region as well as putative tyrosine phosphorylation sites and binding sites for the Src homology 2 domains of the protein tyrosine phosphatase SHP-2 (SH2 domain-containing protein tyrosine phospatase) in its cytoplasmic region (Fujioka et al., 1996; Ohnishi et al., 1996). SHPS-1 is expressed throughout the brain, and the regions in which it is especially abundant overlap in large part with those in which CD47 is also concentrated (Mi et al., 2000; Ohnishi et al., 2005). In addition, we have shown recently that SHPS-1 and CD47 are preferentially localized to axons and dendrites, respectively, in cultured hippocampal neurons (Ohnishi et al., 2005). Together, these observations suggest that CD47 promotes neurite formation and that the interaction of SHPS-1 with CD47 mediates directional intercellular communication between axons and dendrites.

We here examined whether CD47, through its interaction with SHPS-1, indeed promotes dendritic or axonal development in hippocampal neurons in vitro and in vivo. Furthermore, we investigated the intracellular signals that might mediate such actions of CD47 in neurons.

\section{Materials and Methods}

Animals. $C D 47^{+/-}$mice were described previously (Lindberg et al., $1996)$ and were crossed to obtain litters of CD47-deficient $\left(C D 47^{-1-}\right)$ and wild-type (WT) $\left(C D 47^{+/+}\right)$animals. Mice were bred and maintained under specific pathogen-free conditions at the Institute of Experimental Animal Research of Gunma University.

Primary antibodies and reagents. A rat monoclonal antibody $(\mathrm{mAb})$ to mouse CD47 (miap 301) was obtained from PharMingen (San Diego, $\mathrm{CA}$ ). A mouse mAb to the Flag epitope (M2) was from Sigma (St. Louis, $\mathrm{MO}$ ). Rhodamine-conjugated phalloidin was from Invitrogen (Carlsbad, CA), rabbit polyclonal antibodies (pAbs) to C-terminal Src kinase (Csk) were from Santa Cruz Biotechnology (Santa Cruz, CA), and rabbit pAbs to microtubule-associated protein 2 (MAP2) were from Chemicon (Temecula, CA). 4-Amino-5-(4-chlorophenyl)-7-(t-butyl)pyrazolo[3,4d]pyrimidine (PP2) and 4-amino-7-phenylpyrazol[3,4-d]primidine (PP3), a mouse $m A b$ to $\operatorname{Src}(A b-1)$, and a mouse $m A b$ to taul were obtained from Calbiochem (La Jolla, CA). Rabbit pAbs to the Tyr ${ }^{416}$-phosphorylated form of Src were from Cell Signaling Technology (Danver, MA). A mouse mAb to the Myc epitope (9E10) was purified from the culture supernatant of hybridoma cells. Rabbit pAbs to CD47 were prepared as described previously (Miyashita et al., 2004). Rabbit pAbs to mouse FGD1-related Cdc42-guanine nucleotide exchange factor (GEF) (FRG) were generated by injection of rabbits with a synthetic peptide antigen (EPEQSLSPRMQEKHC), of which the sequence corresponded to amino acids $30-40$ of mouse FRG; the pAbs were purified from rabbit serum by affinity chromatography with the peptide antigen conjugated to Sepharose. SHPS-1-Fc was prepared as described previously (Miyashita et al., 2004).

Plasmids. Expression vectors for mouse CD47 form 4 and green fluorescent protein (GFP) $-\beta$-actin were described previously (Miyashita et al., 2004). Plasmids encoding Myc-RacT17N, Myc-neuronal Wiskott-
Aldrich syndrome protein (NWASP)-Cdc42/Rac-interactive binding region (CRIB), Myc-Vav2-WT, Myc-Vav2-dominant negative (DN), Flag-FRG-WT, or Flag-FRG- $\Delta$ DHPH were kindly provided by Y. Takai (Osaka University, Osaka, Japan). A plasmid encoding full-length mouse FRG was obtained from American Type Culture Collection (Manassas, VA) (MGC-6304), and a plasmid encoding myr-Csk, which contains a myristylation signal at its $\mathrm{NH}_{2}$ terminus, was kindly provided by $\mathrm{M}$. Okada (Osaka University). The cDNAs contained in these plasmids were subcloned in the pCAGGS vector, which was kindly provided by J. Miyazaki (Osaka University). Plasmids encoding Raichu-Racl or Raichu-Cdc42 were kindly provided by M. Matsuda (Osaka University).

Determination of dendritic and axonal morphology. Hippocampal neurons $\left(3 \times 10^{5}\right.$ per $35 \mathrm{~mm}$ dish) were isolated from newborn mice [postnatal day 0 (P0) to $\mathrm{P} 1$ ] and plated on culture dishes coated with poly-Dlysine $(25 \mu \mathrm{g} / \mathrm{ml})$ as described previously (Miyashita et al., 2004). The neurons were transfected with expression vectors for GFP-actin and CD47 (as well as with additional expression vectors as indicated) with the use of LipofectAMINE2000 (Invitrogen); transfection was performed the day after plating [ $1 \mathrm{~d}$ in vitro (DIV)] or at 4 DIV. At 3-21 DIV, neurons were fixed for 20-30 min at room temperature with PBS containing $4 \%$ paraformaldehyde and $0.1 \%$ glutaraldehyde and were then permeabilized for $60 \mathrm{~min}$ at room temperature with PBS containing $0.1 \%$ Triton $\mathrm{X}-100$ and $5 \%$ goat serum. They were incubated for $1 \mathrm{~h}$ at room temperature or overnight at $4^{\circ} \mathrm{C}$ with primary antibodies diluted in the permeabilization solution, washed with PBS, and then incubated for $1 \mathrm{~h}$ at room temperature with secondary antibodies conjugated with cyanine 3 (Jackson ImmunoResearch, West Grove, PA), Alexa488 (Invitrogen), or aminomethylcoumarin acetate (Jackson ImmunoResearch). For staining of filamentous actin, neurons were incubated with rhodamineconjugated phalloidin. The cells were finally washed with PBS and examined with a confocal laser-scanning microscope (LSM5 Pascal; Zeiss, Oberkochen, Germany) or an Olympus Optical (Tokyo, Japan) AX-70 microscope. The morphology of transfected neurons was examined with the fluorescence signal of GFP-actin. In other experiments, neurons were cultured at low density $\left(4 \times 10^{4}\right.$ per $35 \mathrm{~mm}$ dish $)$ without forced expression of GFP-actin, and the morphology of dendrites or axons was examined by immunostaining for the dendritic marker MAP2 or the axonal marker taul. The length of axons and dendrites was measured with the use of software of the LSM5 Pascal microscope and was integrated to obtain the total length of these neurites. The number of branches of each axon was quantified by counting the corresponding number of terminal tips.

Quantitation of filopodia and spines. Mouse hippocampal neurons cultured on dishes coated with poly-D-lysine $(25 \mu \mathrm{g} / \mathrm{ml})$ and either control human IgG or SHPS-1-Fc were transfected with expression vectors as described above. Neurons were fixed at 3-21 DIV, and cell morphology was examined by monitoring of GFP-actin fluorescence with the LSM5 Pascal microscope. To quantify the numbers of dendritic filopodia and spines, we classified dendritic protrusions labeled with GFP-actin as filopodia or spines according to the following morphological criteria: (1) a headless protrusion with a length of $>0.5 \mu \mathrm{m}$ and $<8 \mu \mathrm{m}$ and with a length/width ratio of $>2$ was classified as a filopodium; (2) all dendritic protrusions with a length of $>0.5 \mu \mathrm{m}$ and $<8 \mu \mathrm{m}$ other than filopodia were classified as spines.

Immunoprecipitation and immunoblot analysis. Cultured hippocampal neurons $\left(1 \times 10^{6}\right.$ per $35 \mathrm{~mm}$ dish) were washed with ice-cold PBS and then lysed on ice in $1 \mathrm{ml}$ of lysis buffer $(20 \mathrm{~mm}$ Tris- $\mathrm{HCl}, \mathrm{pH}$ 7.6, $140 \mathrm{~mm}$ $\mathrm{NaCl}, 1 \mathrm{~mm}$ EDTA, and 1\% NP-40) containing $1 \mathrm{~mm}$ phenylmethylsulfonyl fluoride, aprotinin $(10 \mu \mathrm{g} / \mathrm{ml})$, and $1 \mathrm{~mm}$ sodium vanadate. The lysates were centrifuged at $21,000 \times g$ for $15 \mathrm{~min}$ at $4^{\circ} \mathrm{C}$, and the resulting supernatants were subjected to immunoprecipitation and immunoblot analysis as described previously (Fujioka et al., 1996; Miyashita et al., 2004).

RNA interference. Two expression vectors, siRNAfrg-GFP\#1 and siRNAfrg-GFP\#2, which each encode an FRG small interfering RNA (siRNA) and enhanced GFP (EGFP), were constructed. Two $19 \mathrm{nt}$ sequences (5'-CTCAGAAACAACTAGTAGA- $3^{\prime}$ and 5'-GGAATTTACTAGCTACTTC-3') corresponding to nucleotides 1138-1156 and 2000-2018 of mouse FRG mRNA (GenBank accession number 
A
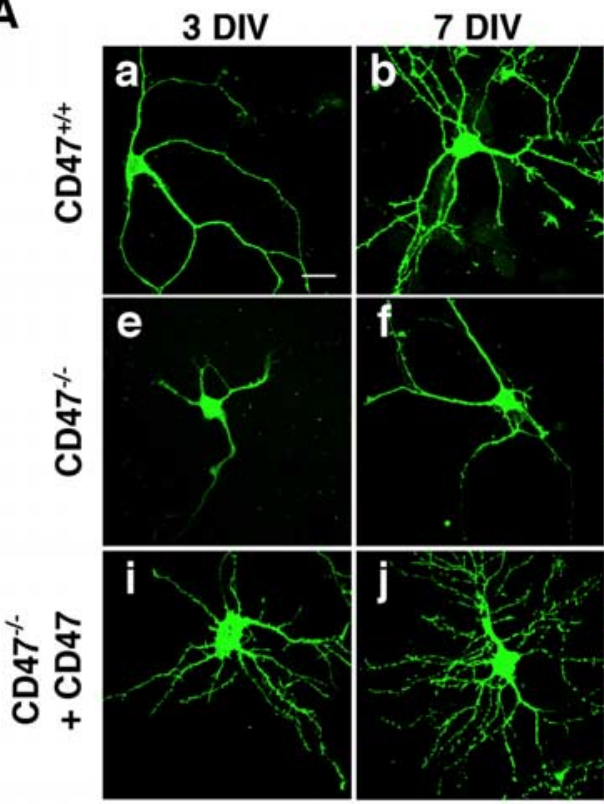

14 DIV
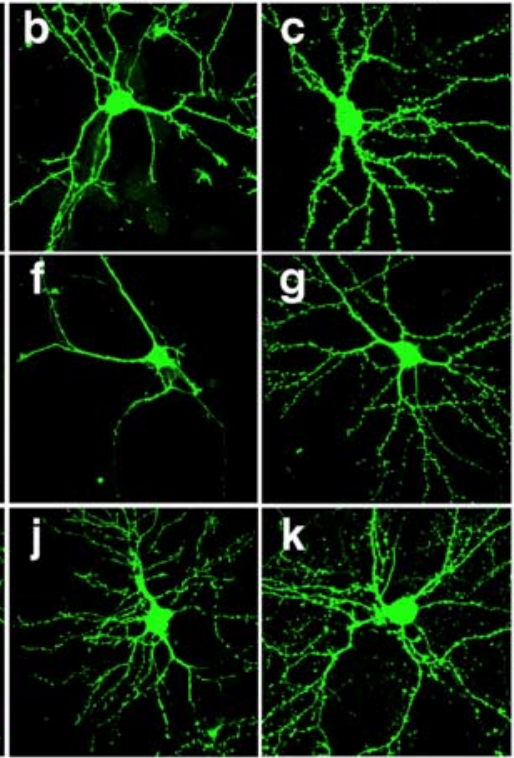

B

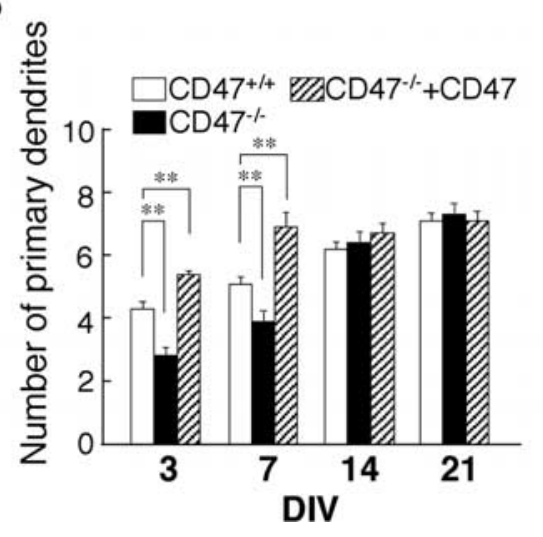

C

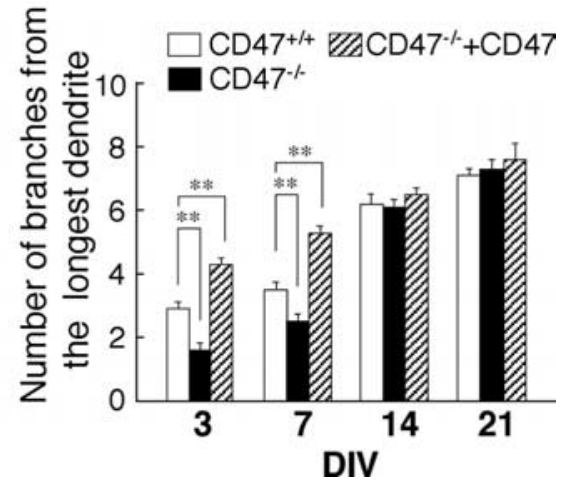

Figure 1. Impairment of dendritic development in cultured hippocampal neurons from $C D 47^{-/-}$mice. $A$, Hippocampal neurons isolated from newborn WT $\left(C D 47^{+/+}\right)(\boldsymbol{a}-\boldsymbol{d})$ or $C D 47^{-/-}(\boldsymbol{e}-\boldsymbol{l})$ mice were transfected with an expression vector for GFP-actin and either an expression vector for CD47 (i-I) or the corresponding empty vector $(\boldsymbol{a}-\boldsymbol{h})$ at 1 DIV $(\boldsymbol{a}, \boldsymbol{e}, \boldsymbol{i})$ or 4 DIV $(\boldsymbol{b}-\boldsymbol{d}$, $\boldsymbol{f}-\boldsymbol{h}, \boldsymbol{j}-\boldsymbol{I})$. Neurons were fixed at 3 DIV $(\boldsymbol{a}, \boldsymbol{e}, \boldsymbol{i}), 7 \mathrm{DIV}(\boldsymbol{b}, \boldsymbol{f}, \boldsymbol{j}), 14 \mathrm{DIV}(\boldsymbol{c}, \boldsymbol{g}, \boldsymbol{k})$, or $21 \mathrm{DIV}(\boldsymbol{d}, \boldsymbol{h}, \boldsymbol{I})$, and the morphology of each neuron as revealed by GFP-actin was examined by fluorescence microscopy. Scale bar, $20 \mu \mathrm{m}$. $\boldsymbol{B}, \boldsymbol{C}$, Neurons treated as in $\boldsymbol{A}$ were evaluated for their dendritic morphology at the indicated times. The numbers of primary dendrites per neuron $(\boldsymbol{B})$ and of branches from the longest primary dendrite of each neuron ( $C$ were determined for $C D 47^{+/+}$neurons (open columns), $C D 47^{-/-}$neurons (filled columns), and $C D 47^{-1-}$ neurons expressing ectopic CD47 (hatched columns). Data are means \pm SE of values obtained from a total of $28-36$ neurons in three independent experiments. ${ }^{* *} p<0.01$ (Student's $t$ test) for the indicated comparisons.

NM145519) were selected for construction of the siRNAfrg-GFP vectors, which direct the synthesis of the corresponding 19 bp double-stranded target sequence. Two pairs of $64 \mathrm{nt}$ sequences, each of which contains a targeting sequence and its reverse complementary sequence (pair 1, $5^{\prime}$ GATCCCCCTCAGAAACAACTAGTAGATTCAAGAGATCTACTAGTTGTTTCTGAGTTTTTGGAAA- ${ }^{\prime}$ and 5'-AGCTTTTCCAAAAACTCAGAAACAACTAGTAGATCTCTTGAATCTACTAGTTGTTTCTGAGGGG-3'; pair 2, 5' -GATCCCCGGAATTTACTAGCTACTTCTTCAAGAGAGAAGTAGCTAGTAAATTCCTTTTTGGAAA- ${ }^{\prime}$ and $5^{\prime}$-AGCTTTTCCAAAAAGGAATTTACTAGCTACTTCTCTCTTGAAGAAGTAGCTAGTAAATTCCGGG-3'), were synthesized. After an annealing step, each pair of oligonucleotides was inserted into the PSUPER vector (Brummelkamp et al., 2002). The resulting vectors, pSUPER-mFRG\#1 and pSUPER-mFRG\#2, were digested with KpnI and SacI, and the released DNA fragment containing the $\mathrm{H} 1$ promoter and downstream short-hairpin sequence was rendered blunt-ended and subcloned into the blunted SalI site of the EGFP expression vector pCAGGS-
EGFP. The resulting vectors were designated siRNAfrg-GFP\#1 and siRNAfrg-GFP\#2.

Fluorescent resonance energy transfer analysis. The fluorescence resonance energy transfer (FRET) imaging was performed as described previously (Itoh et al., 2002). In brief, primary cultured mouse hippocampal neurons were cotransfected with expression vectors for CD47 (or the corresponding empty vector) and either Raichu-Rac1 or Raichu-Cdc42 at 1 DIV. Forty-eight hours after transfection (at 3 DIV), the cells were fixed and stained with $\mathrm{mAb}$ to CD47 to confirm the expression of exogenous CD47. FRET images were acquired on the Aquacosmos/Ashura system (Hamamatsu Photonics, Shizouka, Japan) using an IX-81 inverted microscope equipped with a UPlanApo $100 \times, 1.35$ numerical aperture oil-immersion objective (Olympus Optical), a 440AF21 excitation filter, a 455DRLP dichroic mirror, a 460ALP emission filter, and a three CCD color camera (C7780-22; Hamamatsu Photonics). Image acquisition and analysis were performed using Aquacosmos 2.6 software (Hamamatsu Photonics).

\section{Results}

Impaired development of dendrites and axons in hippocampal neurons of CD47-deficient mice

We have shown recently that the abundance of CD47 in dendrites is greater than that in axons of cultured hippocampal neurons (Ohnishi et al., 2005). To evaluate the possible role of $\mathrm{CD} 47$ in the regulation of neuronal development, we prepared cultures of hippocampal neurons from $\mathrm{WT}$ and $C D 47^{-1-}$ mice (Lindberg et al., 1996) and then determined the numbers of primary dendrites, which are derived directly from the cell body, in these neurons.

As described previously (Ohnishi et al., 2005), the expression of CD47 in hippocampal neurons isolated from newborn WT mice was apparent at only a low level after 3 DIV but had increased markedly by 7 DIV (see Fig. 3Ae,Ag). Such expression was not detected in neurons prepared from $C D 47^{-/-}$mice (supplemental Fig. 1, available at www.jneurosci.org as supplemental material). To examine the morphology of individual neurons in detail, we transfected the cells with an expression vector for a fusion protein containing GFP and $\beta$-actin at 1 or 4 DIV and then cultured them further for various times (Fig. 1). The number of primary dendrites on each $C D 47^{-1-}$ neuron at 3 or 7 DIV was markedly smaller than that apparent for WT neurons (Fig. 1A). Quantitative analysis revealed that this difference was statistically significant $($ Fig. $1 B$ ). For this analysis, a primary dendrite was defined as a process derived directly from the cell body with a length equal to or greater than one-half of the diameter of the cell body $(\sim 8 \mu \mathrm{m})$ and with a diameter of $>2 \mu \mathrm{m}$.

We also examined the effect of CD47 deficiency on the branching of dendrites by counting the total number of branches derived from the longest primary dendrite of each neuron. A 
A
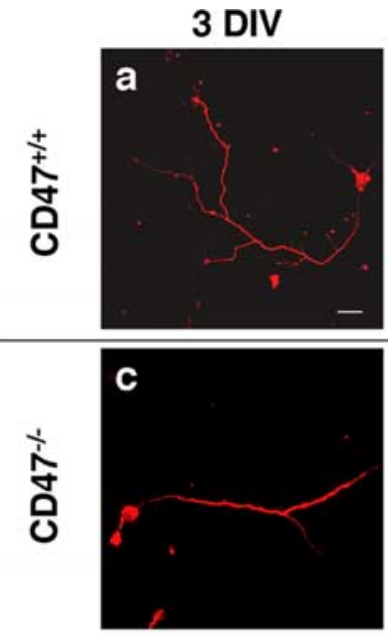

B

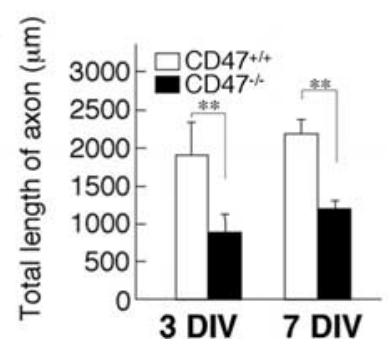

7 DIV
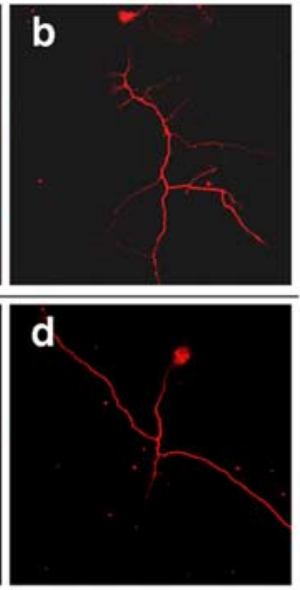

C

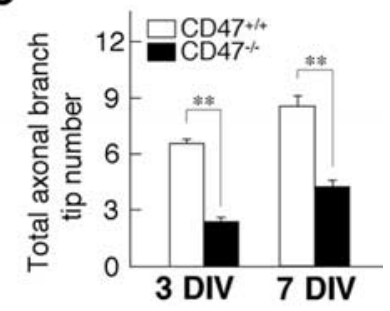

Figure 2. Impairment of axonal development in cultured hippocampal neurons from $C D 47^{-1-}$ mice. $A$, Cultured hippocampal neurons from $C D 47^{+/+}(\boldsymbol{a}, \boldsymbol{b})$ or $C D 47^{-1-}(\boldsymbol{c}, \boldsymbol{d})$ mice were fixed at 3 DIV $(\boldsymbol{a}, \boldsymbol{c})$ or 7 DIV $(\boldsymbol{b}, \boldsymbol{d})$ and stained with an mAb to tau1. Scale bar, $20 \mu \mathrm{m} . \boldsymbol{B}$, $\boldsymbol{C}$, The length of the primary axon $(\boldsymbol{B})$ and the number of terminal branch tips for the primary axon $(\boldsymbol{C})$ were determined for neurons cultured and stained as in $\boldsymbol{A}$. Data are means \pm SE of values obtained from a total of 28 neurons in three independent experiments. ${ }^{* *} p<0.01$ (Student's $t$ test).

branch was defined as a process whose length was at least equal to one-half of the diameter of the cell body. Quantitative analysis revealed that the number of branches originating from the longest primary dendrite of $C D 47^{-1-}$ neurons was also significantly decreased at 3 or 7 DIV compared with that apparent for WT neurons (Fig. 1C). In contrast, the number of primary dendrites or that of branches from the longest primary dendrite did not differ between $C D 47^{-/-}$and WT neurons at 14 or 21 DIV (Fig. 1).

We also examined the morphology of hippocampal neurons cultured at low density without transfection and stained with pAbs to MAP2, a dendritic marker protein. We measured the total length of all dendrites and their branches in the neurons at 3 and 7 DIV. The total length of dendrites was markedly reduced in $C D 47^{-1-}$ neurons compared with WT neurons at both time points (supplemental Fig. $2 A, B$, available at www.jneurosci.org as supplemental material). The numbers of primary, secondary, and tertiary dendritic branches were also markedly reduced in $C D 47^{-1-}$ neurons at 3 or 7 DIV compared with those for WT neurons (supplemental Fig. 2A, C, available at www.jneurosci. org as supplemental material). In addition, the number of branches from the longest primary dendrite was also substantially decreased in $C D 47^{-/-}$neurons at either 3 or 7 DIV compared with that for WT neurons (data not shown).

We next examined the effect of CD47 deficiency on axon morphology by staining cultured hippocampal neurons with an $\mathrm{mAb}$ to taul, an axon marker protein (Fig. 2). The length of the primary axon of $C D 47^{-/-}$neurons was significantly smaller than that for WT neurons at 3 or 7 DIV (Fig. $2 A, B$ ). We also counted the number of terminal tips derived from each primary axon to determine the extent of axonal branching; this parameter was also markedly reduced for $C D 47^{-1-}$ neurons at 3 or 7 DIV compared with that for WT neurons (Fig. 2C). In addition, the percentage of neurons that manifested an enlarged growth cone (a growth cone with four or more filopodia and with lamellipodia; other growth cones were considered to be collapsed) at the most distal end of the primary axon was significantly smaller for $C D 47^{-1-}$ neurons $(45.2 \pm 5.09 \% ; n=500$ neurons) than for WT neurons $(63.8 \pm 6.91 \% ; n=500$ neurons; $p=0.014$, Student's $t$ test) at 4 DIV. Together, these data suggested that CD47 is required for proper development of both dendrites and axons in cultured hippocampal neurons during the first few (3-7) DIV. However, it may not be required for additional development of dendrites at later stages (14-21 DIV).

\section{Effects of forced expression of CD47 on dendrite development}

The development of dendrites and axons was markedly impaired in hippocampal neurons of $C D 47^{-/-}$mice. We therefore next examined the effect of forced expression of CD47 on the morphology of hippocampal neurons of WT mice. For these experiments, we focused on the contribution of CD47 to dendritic development because the morphology of dendrites is more readily determined than is that of axons, which are highly elongated and manifest multiple and complex branching patterns. One of four alternatively spliced isoforms of CD47 (form 4) predominates in the brain (Reinhold et al., 1995). We therefore transfected hippocampal neurons cultured at high density with expression vectors for mouse CD47 (form 4) and GFP-actin at 1 or 4 DIV and examined the morphology of the neurons at 3-21 DIV. The expression of endogenous CD47 in hippocampal neurons from WT mice was detected at a relatively low level at $3 \mathrm{DIV}$ (Fig. 3Ae). Transfection with the CD47 vector resulted in marked increases in both the abundance of this protein (Fig. $3 A f, A h$ ) and the number of primary dendrites apparent at 3 or 7 DIV (Fig. $3 A, B$ ). Forced expression of CD47 also increased the number of branches from the longest primary dendrite at these time points (Fig. 3C). However, the number of primary dendrites or that of branches from the longest primary dendrite did not differ between the neurons overexpressing CD47 and mock-transfected neurons at 14 or 21 DIV (Fig. $3 B, C$ ).

We also examined the effect of forced expression of CD47 on dendrite development in $C D 47^{-1-}$ neurons. Expression of CD47 in $C D 47^{-1-}$ neurons reversed the decreases in the numbers of primary dendrites and of branches from the longest primary dendrite apparent in these cells at 3 or 7 DIV (Fig. $1 A i-A l$ ); indeed, these parameters in the $C D 47^{-/-}$neurons expressing CD47 were significantly increased compared with those for WT neurons (Fig. $1 B, C$ ).

Effects of an SHPS-1-Fc fusion protein and forced expression of CD47 on the formation of dendritic filopodia and spines

The development of dendrites is accompanied by the extension of filopodia, which form contacts with synaptic terminals of axons and then mature into dendritic spines (Ziv and Smith, 1996). We have shown previously that SHPS-1-Fc, an Fc fusion protein containing the extracellular region of the CD47 ligand SHPS-1, promoted filopodium formation in N1E-115 neuroblastoma cells and that this effect was enhanced by overexpression of CD47 (Miyashita et al., 2004). We therefore next examined the effects of SHPS-1-Fc and forced expression of CD47 on the formation of 

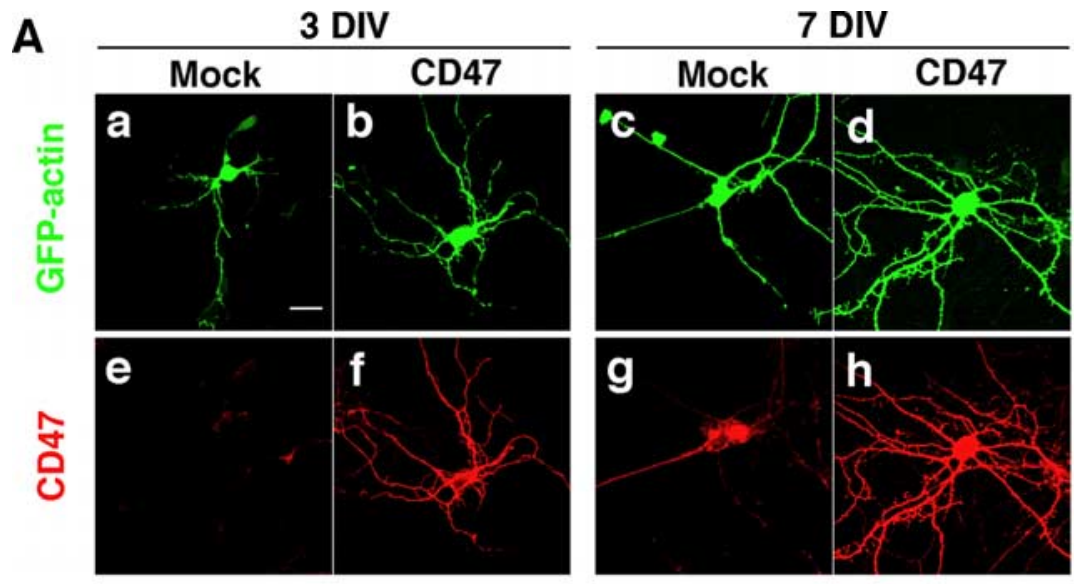

B

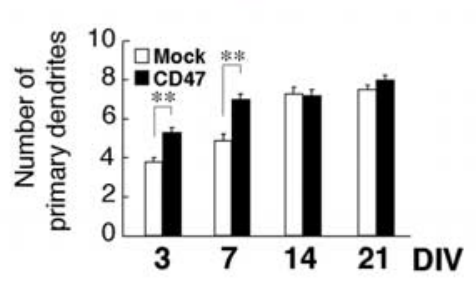

D

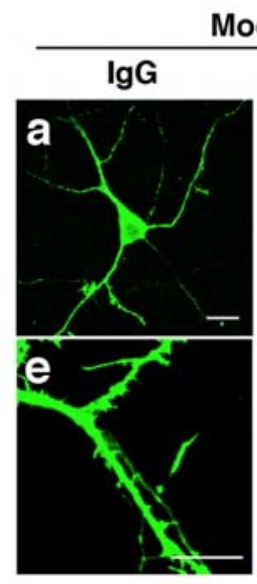

Mock
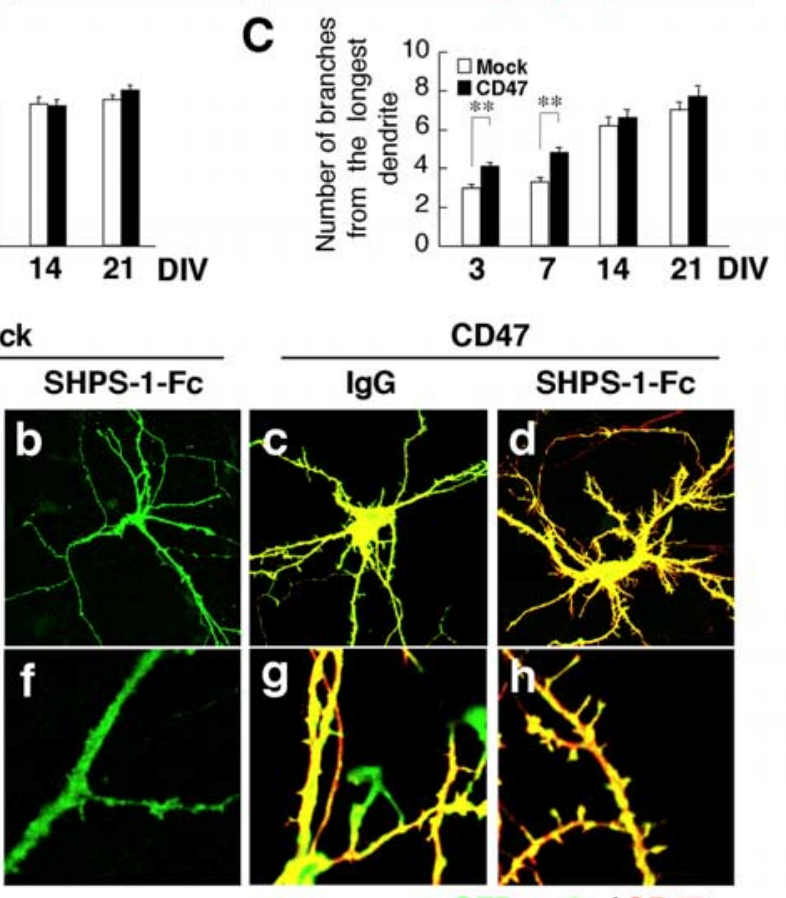

E

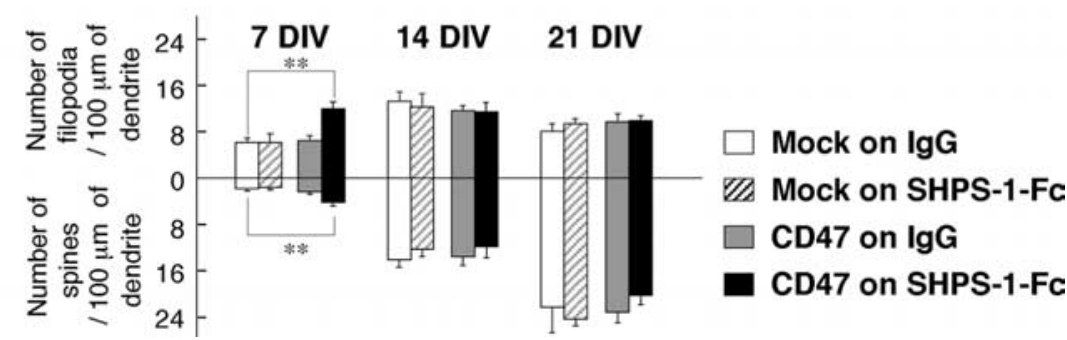

Figure 3. Effects of SHPS-1-Fc and forced expression of CD47 on dendrite development and the formation of filopodia and spines. $\boldsymbol{A}$, Hippocampal neurons from WT mice were transfected with an expression vector for GFP-actin and either a vector for CD47 $(\boldsymbol{b}, \boldsymbol{d}, \boldsymbol{f}, \boldsymbol{h})$ or the corresponding empty vector $(\boldsymbol{a}, \boldsymbol{c}, \boldsymbol{e}, \boldsymbol{g})$ at 1 DIV $(\boldsymbol{a}, \boldsymbol{b}, \boldsymbol{e}, \boldsymbol{f})$ or 4 DIV $(\boldsymbol{c}, \boldsymbol{d}, \boldsymbol{g}, \boldsymbol{h})$. Neurons were fixed at 3 DIV $(\boldsymbol{a}, \boldsymbol{b}, \boldsymbol{e}, \boldsymbol{f})$ or 7 DIV $(\boldsymbol{c}, \boldsymbol{d}, \boldsymbol{g}, \boldsymbol{h})$ and stained with an mAb to CD47 $(\boldsymbol{e}-\boldsymbol{h})$. Fluorescence images of GFP-actin were also obtained $(\boldsymbol{a}-\boldsymbol{d})$. Scale bar, $20 \mu \mathrm{m} . \boldsymbol{B}, \boldsymbol{C}$, The number of primary dendrites per neuron $(\boldsymbol{B})$ and that of branches from the longest primary dendrite of each neuron $(\boldsymbol{C})$ were determined for cells treated as in $\boldsymbol{A}$ and cultured for the indicated times. Data are means \pm SE of values obtained from a total of $22-30$ neurons in three independent experiments. ${ }^{* *} p<0.01$ (Student's $t$ test). $\boldsymbol{D}$, Hippocampal neurons from WT mice were cultured on dishes coated with SHPS-1-Fc $(\boldsymbol{b}, \boldsymbol{d}, \boldsymbol{f}, \boldsymbol{h})$ or control human $\lg G(\boldsymbol{a}, \boldsymbol{c}, \boldsymbol{e}, \boldsymbol{g})$ and were transfected at 4 DIV with a vector for GFP-actin and either a vector for CD47 $(\boldsymbol{c}, \boldsymbol{d}, \boldsymbol{g}, \boldsymbol{h})$ or the corresponding empty vector $(\boldsymbol{a}, \boldsymbol{b}, \boldsymbol{e}$, f). Neurons were fixed and stained with an mAb to CD47 (red) as well as monitored for GFP-actin fluorescence (green) at 7 DIV. Scale bar, $20 \mu \mathrm{m}$. $\boldsymbol{E}$, The densities of dendritic filopodia and spines at 7, 14, and 21 DIV were determined for neurons treated as in D. Data are means \pm SE of values obtained from a total of $18-24$ neurons in three independent experiments. ${ }^{* *} p<0.01$ (Student's $t$ test). dendritic filopodia and spines in hippocampal neurons of WT mice.

We classified dendritic protrusions labeled with GFP-actin as filopodia or spines on the basis of morphological characteristics proposed in a previous study (Takahashi et al., 2003) and as described in Materials and Methods. At 7 DIV, the number of dendritic filopodia was greater than that of spines (Fig. 3D,E). The numbers of these structures were approximately equal at $14 \mathrm{DIV}$, and the number of spines was greater than that of filopodia at 21 DIV (Fig. 3E), consistent with previous observations (Takahashi et al., 2003). Neither SHPS-1-Fc nor forced expression of CD47 alone affected the formation of filopodia or spines at 7-21 DIV (Fig. $3 D, E)$. In contrast, the combination of SHPS-1-Fc and forced expression of CD47 resulted in a significant increase in the numbers of both filopodia and spines at 7 DIV compared with those apparent for mock-transfected neurons plated on control human IgG (Fig. $3 D, E$ ). Such effects were not apparent at 14 or 21 DIV (Fig. 3E). We also examined the effects of SHPS-1-FC and forced expression of CD47 on dendrite development in cultured hippocampal neurons. SHPS-1-Fc alone failed to increase the number of primary dendrites or that of branches from the longest primary dendrite in mocktransfected neurons at 7 DIV (supplemental Fig. 3, available at www.jneurosci.org as supplemental material). In addition, SHPS-1-Fc did not further increase the number of primary dendrites or that of branches from the longest primary dendrite in CD47-overexpressing neurons at 7 DIV (supplemental Fig. 3, available at www.jneurosci.org as supplemental material).

\section{Role of Rac and Cdc42 in dendritic} development promoted by CD47

We next investigated the intracellular signaling pathway responsible for the stimulatory effect of CD47 on dendritic development. Hippocampal neurons from WT mice were transfected with expression vectors for GFP-actin, CD47, and either a dominant-negative mutant of Racl (RacT17N) or the CRIB domain of NWASP (NWASP-CRIB) at 4 DIV and were examined for their morphology at 7 DIV. Immunostaining confirmed that individual neurons were successfully transfected with the combination of three different plasmids (supplemental Fig. 4, available at www.jneurosci.org as supplemental material). Forced expression of Myc epitope-tagged RacT17N in the ab- 
A
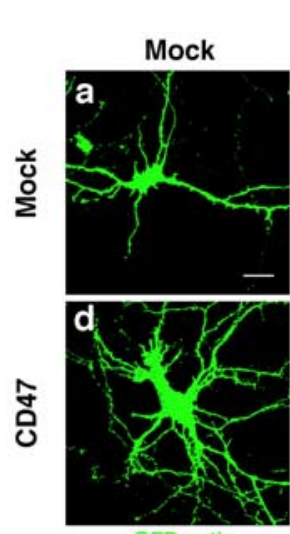

GFP-actin

B

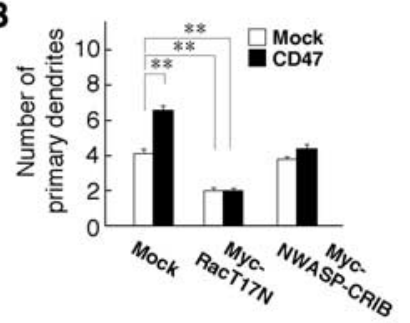

C

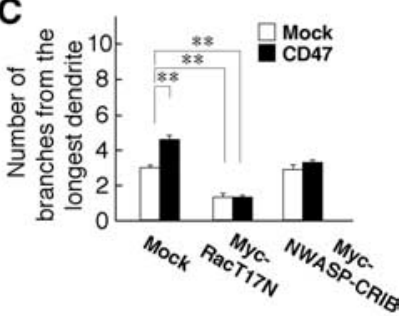

D

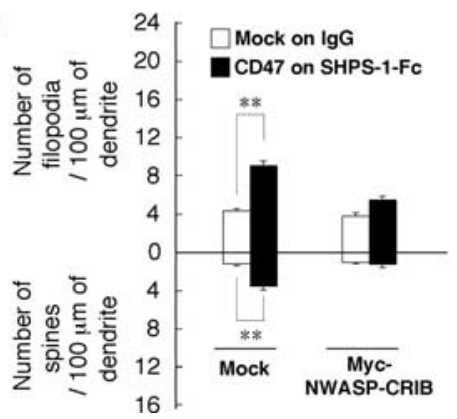

Figure 4. Participation of Rac and Cdc42 in the promotion of dendritic development by forced expression of CD47. A, Hippocampal neurons from WT mice were transfected at 4 DIV with an expression vector for GFP-actin, a CD47 vector $(\boldsymbol{d}-\boldsymbol{f})$ or the corresponding empty vector $(\boldsymbol{a}-\boldsymbol{c})$, and either a vector for Myc epitope-tagged RacT17N $(\boldsymbol{b}, \boldsymbol{e})$ or Myc-NWASP-CRIB $(\boldsymbol{c}, \boldsymbol{f})$ or the corresponding empty vector $(\boldsymbol{a}, \boldsymbol{d})$. At 7 DIV, the neurons were fixed and stained with an $\mathrm{mAb}$ to Myc (red), and GFP-actin fluorescence was also monitored (green). Neurons were also stained with an $\mathrm{mAb}$ to $\mathrm{CD} 47$ to confirm its overexpression (data not shown; see supplemental Fig. 3, available at www.jneurosci.org as supplemental material). Scale bar, $20 \mu \mathrm{m}$. B, C, The number of primary dendrites per neuron $(\boldsymbol{B})$ and that of branches from the longest primary dendrite of each neuron $(\boldsymbol{C})$ were determined for neurons treated as in $\boldsymbol{A}$. Data are means $\pm \mathrm{SE}$ of values obtained from a total of 30 neurons in three independent experiments. ${ }^{* *} p<0.01$ (Student's $t$ test). D, Hippocampal neurons from WT mice were plated on dishes coated with SHPS-1-Fc or control human IgG and were transfected at 4 DIV with a vector for GFP-actin, a vector for CD47 or the corresponding empty vector, and a vector for Myc epitope-tagged NWASP-CRIB or the corresponding empty vector. At 7 DIV, the neurons were fixed and stained with an $\mathrm{mAb}$ to Myc, and the densities of dendritic filopodia and spines were determined. Data are means $\pm S E$ of values obtained from a total of 30 neurons in three independent experiments. ${ }^{* *} p<0.01$ (Student's $t$ test).

sence of that of CD47 resulted in marked increases in the diameter of primary dendrites and in the formation of filopodia at the margin of these dendrites (Fig. 4Ab). Furthermore, expression of RacT17N prevented the increases in both the number of primary dendrites and branches from the longest primary dendrite induced by forced expression of CD47, although it also reduced these parameters in neurons not overexpressing CD47 (Fig. $4 A b, A e, B, C)$. NWASP-CRIB specifically binds the GTP-bound
A

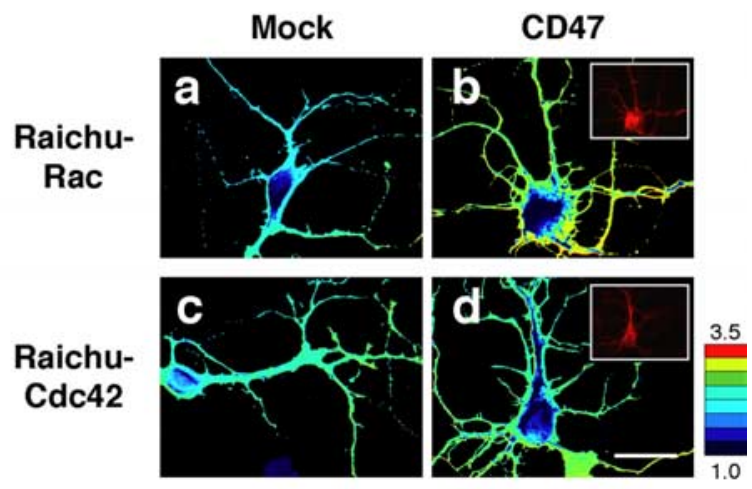

B

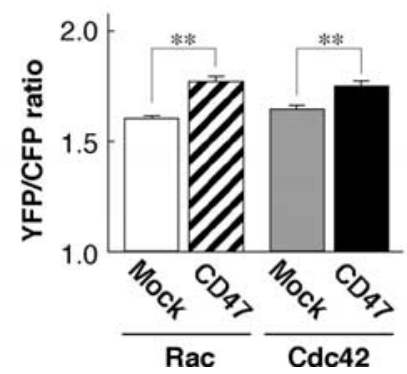

Figure 5. Activation of Rac and Cdc 42 by forced expression of CD47 in hippocampal neurons. $\boldsymbol{A}$, Hippocampal neurons from WT mice were transfected with an expression vector for CD47 (b $\boldsymbol{d}$ ) (or the corresponding empty vector (Mock; $\boldsymbol{a}, \boldsymbol{c}$ ) and a vector for either Raichu-Rac $(\boldsymbol{a}, \boldsymbol{b})$ or Raichu-Cdc42 (c, $\boldsymbol{d})$ at 1 DIV. Neurons were fixed at 3 DIV and stained with mAb to CD47 to confirm the expression of CD47 (shown in insets in $\boldsymbol{b}$ and $\boldsymbol{d}$ ). The images of YFP/CFP ratio of transfected neurons were shown in the intensity-modulated display mode. In the intensitymodulated display mode, eight colors from red to blue are used to represent the YFP/CFP ratio, with each color indicating the mean intensity of YFP/CFP ratio. The upper and lower limits of the ratio image are shown on the right. Scale bar, $20 \mu \mathrm{m}$. B, YFP/CFP ratios in dendrites were quantified in transfected neurons. Data are means \pm SE of values from eight neurons and are representative of three independent experiments. ${ }^{* *} p<0.01$ (Student's $t$ test).

(active) form of Cdc42 and thereby inhibits its activity (Miyashita et al., 2004). Expression of a Myc epitope-tagged form of NWASP-CRIB also prevented the effects of ectopic CD47 on the numbers of primary dendrites and branches from the longest primary dendrite, although it did not affect these parameters in neurons not overexpressing CD47 (Fig. $4 A c, A f, B, C$ ). In addition, expression of NWASP-CRIB prevented the increase in the density of dendritic filopodia and spines apparent in neurons overexpressing CD47 and plated on SHPS-1-Fc (Fig. 4D).

We next determined whether forced expression of CD47 could promote the activation of Rac or Cdc42 in hippocampal neurons by the use of FRET imaging, which used the FRET probes Raichu-Rac and Raichu-Cdc42 (Itoh et al., 2002). The FRET imaging showed marked increases of the values of yellow fluorescent protein (YFP)/cyan fluorescent protein (CFP) ratio, with both Raichu-Rac and Raichu-Cdc42, at dendrites from CD47-expressing hippocampal neurons compared with that apparent with control mock-transfected neurons (Fig. $5 A, B)$. These results suggest that forced expression of CD47 indeed induces activation of both Rac and $\mathrm{Cdc} 42$ in hippocampal neurons. 
A
Mock

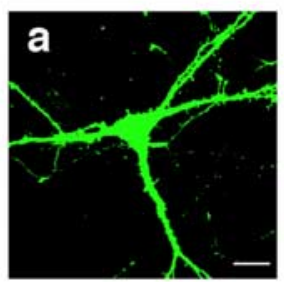

B

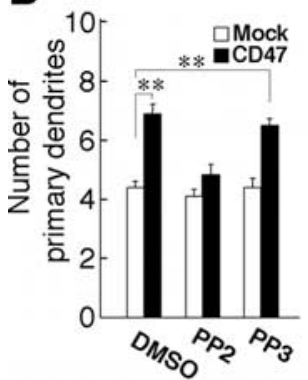

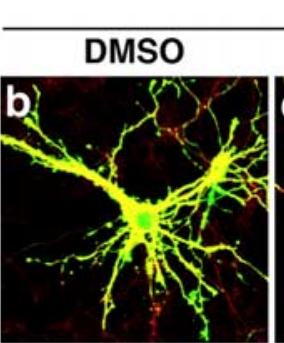

C

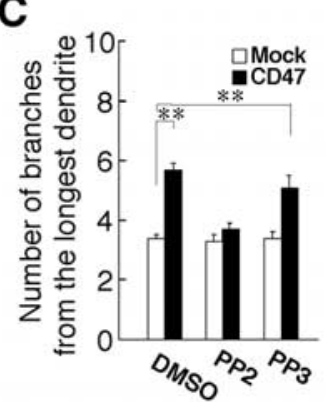

CD47

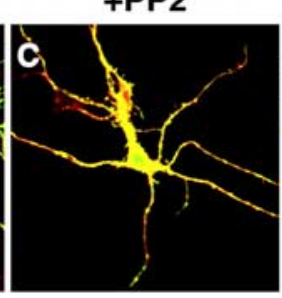

D
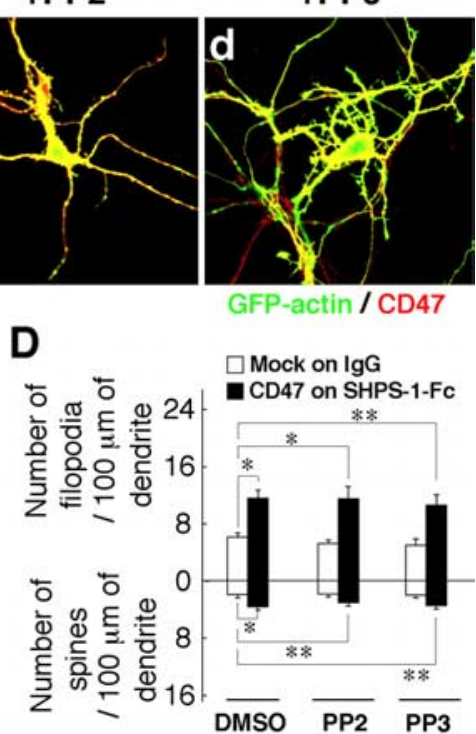

GFP-actin / CD47
E

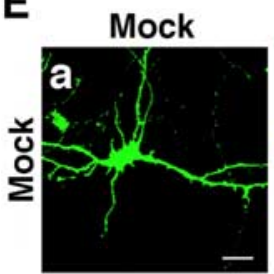

\section{myr-Csk}

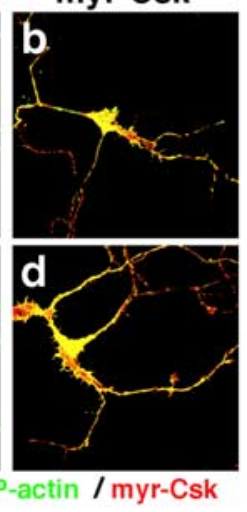

F

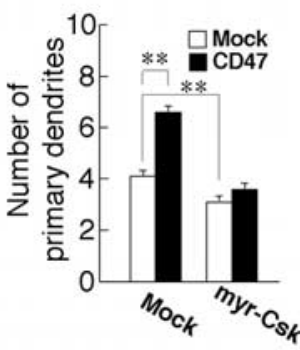

G

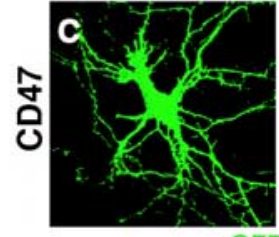

GFP-actin / myr-Csk

H
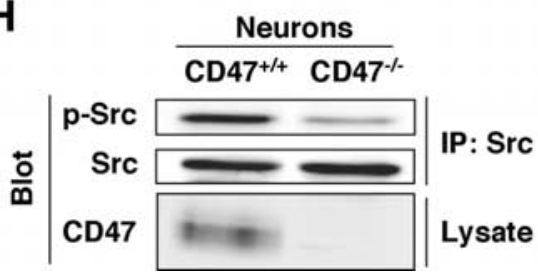

I

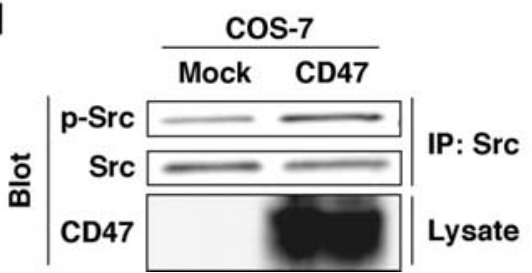

Figure 6. Participation of an Src family kinase in the promotion of dendritic development by CD47. A, Hippocampal neurons from WT mice were transfected at 4 DIV with an expression vector for GFP-actin and either a vector for CD47 $(\boldsymbol{b}-\boldsymbol{d})$ or the corresponding empty vector $(\boldsymbol{a})$. They were then cultured in the presence of $20 \mu \mathrm{m} \mathrm{PP2}(\boldsymbol{c}), 20 \mu \mathrm{M} \mathrm{PP3}(\boldsymbol{d})$, or dimethylsulfoxide (DMSO) vehicle $(\boldsymbol{a}, \boldsymbol{b})$. At 7 DIV, neurons were fixed and stained with an $\mathrm{mAb}$ to CD47 (red), and GFP-actin fluorescence was monitored (green). Scale bar, $20 \mu \mathrm{m}$. $\boldsymbol{B}-\boldsymbol{D}$, The number of primary dendrites per neuron $(\boldsymbol{B})$, that of branches from the longest primary dendrite of each neuron $(\boldsymbol{C})$, and the density of dendritic filopodia or spines $(\boldsymbol{D})$ were determined for neurons transfected and treated with PP2 or PP3 as in $\boldsymbol{A}$. In $\boldsymbol{D}$, neurons transfected with the CD47 vector were plated on SHPS-1-Fc, and those transfected with the corresponding empty vector were plated on human lgG. Data are means \pm SE of values obtained from a total of 33-36 neurons $(\boldsymbol{B}, \boldsymbol{C})$ or $28-32$ neurons $(\boldsymbol{D})$ in three independent experiments. ${ }^{*} p<0.05,{ }^{* *} p<0.01$ (Student's $t$ test). $\boldsymbol{E}$, Hippocampal neurons from WT mice were transfected at 4 DIV with an expression vector for GFP-actin, with either a vector for myr-Csk $(\boldsymbol{b}, \boldsymbol{d})$ or the corresponding empty vector $(\boldsymbol{a}, \boldsymbol{c})$, and with either a vector for $\operatorname{CD} 47(\boldsymbol{c}, \boldsymbol{d})$ or the corresponding empty vector $(\boldsymbol{a}, \boldsymbol{b})$. At 7 DIV, neurons were fixed and stained with pAbs to Csk (red), and GFP-actin fluorescence was monitored (green). Neurons were also stained with an mAb to CD47 to confirm its expression (data not shown). Scale bar, $20 \mu \mathrm{m}$. F, G, The number of primary dendrites per neuron $(\boldsymbol{F})$ and that of branches from the longest primary dendrite of each neuron $(\boldsymbol{G})$ were determined for neurons treated as in $\boldsymbol{E}$. Data are means $\pm \mathrm{SE}$ of values obtained from a total of 30 neurons in three independent experiments. ${ }^{* *} p<0.01$ (Student's $t$ test). $\boldsymbol{H}$, Cultured hippocampal neurons from $\mathrm{CD} 47^{+/+}$or $\mathrm{CD} 7^{-/-}$mice were lysed at 7 DIV and subjected to immunoprecipitation (IP) with an $\mathrm{mAb}$ to $\mathrm{c}-\mathrm{Src}$. The resulting precipitates were subjected to immunoblot analysis both with pAbs to the $\mathrm{Tyr}^{416}$-phosphorylated form of $\mathrm{c}-\mathrm{Src}(\mathrm{p}-\mathrm{Src})$ and with the mAb to c-Src. Cell lysates were also directly subjected to immunoblot analysis with pAbs to CD47. Data are representative of three independent experiments. I, COS-7 cells

Role of an Src family kinase in dendritic development promoted by CD47

We next continued our attempt to identify downstream signaling molecules responsible for the promotion of dendritic development in hippocampal neurons by CD47. Treatment of neurons with PP2, an inhibitor of Src family kinases (Hanke et al., 1996), prevented the increases in the number of primary dendrites per neuron and branches from the longest primary dendrite induced by forced expression of CD47 in hippocampal neurons from WT mice; it did not affect these parameters in neurons not overexpressing CD47 (Fig. $6 A a-A c, B, C)$. In addition, PP3, an inactive analog of PP2, did not inhibit these effects of CD47 (Fig. 6Ad, B,C). In contrast, PP2 and PP3 each failed to inhibit the formation of dendritic filopodia and spines induced by overexpression of CD47 and exposure to SHPS-1-Fc (Fig. 6D).

Csk inhibits the activity of Src family kinases by phosphorylating their $\mathrm{COOH}$ terminal tyrosine residue (Honda et al., 1997). In addition, a membrane-targeted form of Csk (myr-Csk), which contains a myristylation signal at its $\mathrm{NH}_{2}$ terminus, has been shown to inhibit the activity of Src family kinases more effectively than does wild-type Csk (Honda et al., 1997). Forced expression of myr-Csk prevented the increases in the number of primary dendrites per neuron and in the number of branches from the longest primary dendrite induced by forced expression of $\mathrm{CD} 47$, whereas it also reduced these parameters in neurons not overexpressing CD47 (Fig. 6E-G).

We then examined the effect of CD47 on the tyrosine phosphorylation state and activation of Src in hippocampal neurons. Activation of c-Src results in autophosphorylation of the enzyme on tyrosine416 (Brown and Cooper, 1996). Immunoprecipitates prepared from lysates of WT or $C D 47^{-/-}$hippocampal neurons with an $\mathrm{mAb}$ to c-Src were subjected to immunoblot analysis with pAbs to the $\mathrm{Tyr}^{416}$ phosphorylated form of c-Src. The phosphorylation of c-Src on $\mathrm{Tyr}^{416}$ was markedly reduced in $C D 47^{-/-}$neurons compared with that in WT neurons (Fig. $6 \mathrm{H}$ ). Furthermore, forced expression of CD47 in COS-7 cells resulted in an in-

\section{$\leftarrow$}

were transfected with a vector for CD47 or the corresponding empty vector. Twenty-four hours after transfection, the cells were lysed and subjected to immunoprecipitation with an $\mathrm{mAb}$ to $\mathrm{c}-\mathrm{Src}$. The resulting precipitates as well as the original cell lysates were then subjected to immunoblot analysis as in $\boldsymbol{H}$. Data are representative of three independent experiments. 
crease in the phosphorylation of c-Src on $\mathrm{Tyr}^{416}$ (Fig. 6I). Together, these data suggested that an Src family kinase mediates, at least in part, the promotion of dendritic development in hippocampal neurons by CD47.

\section{Role of Vav2 and FRG in the promotion of dendritic development by CD47}

Vav2, a GEF for Rho family proteins, is activated by Src-mediated tyrosine phosphorylation (Marignani and Carpenter, 2001; Servitja et al., 2003). In addition, FRG, another GEF specific for Cdc42 and Rac, undergoes tyrosine phosphorylation by c-Src and is thereby activated (Miyamoto et al., 2003; Fukuhara et al., 2004). Immunoblot analysis has revealed that both Vav2 and FRG are expressed in cultured hippocampal neurons (Kubo et al., 2002) (data not shown). We thus next examined whether Vav2 or FRG participates in CD47-induced dendritic development.

We first investigated the effects of forced expression of a dominant-negative mutant of Vav2 (Vav2-DN), in which Leu ${ }^{212}$ is replaced by Gln (Kodama et al., 2000; Kawakatsu et al., 2005). Forced expression of Vav2-DN, but not that of Vav2-WT, prevented the increases in both the number of primary dendrites per neuron and the number of branches from the longest primary dendrite induced by forced expression of CD47; it had no effect on these parameters in neurons not overexpressing CD47 (Fig. $7 A-C)$. In contrast, forced expression of neither Vav2-DN nor Vav2-WT inhibited the increase in the density of dendritic filopodia and spines induced by CD47 overexpression and exposure to SHPS-1-Fc (Fig. 7D).

We next examined the effects of forced expression of a dominant-negative mutant of FRG (FRG- $\Delta$ DHPH), which lacks both the DH and PH domains of the intact protein (Miyamoto et al., 2003; Fukuhara et al., 2004). Expression of FRG- $\Delta$ DHPH prevented the increases in both the number of primary dendrites per neuron and the number of branches from the longest primary dendrite induced by forced expression of CD47; it had no effect on these parameters in neurons not overexpressing CD47 (supplemental Fig. 5Ab, $A e, B, C$, available at www.jneurosci.org as supplemental material). However, forced expression of FRG-WT also prevented these effects of CD47 (supplemental Fig. $5 A f, B, C$ ). Forced expression of FRG-WT was shown previously to prevent neurite extension by cortical neurons (Kubo et al., 2002). We therefore examined the effect of depletion of endogenous FRG by RNA interference (RNAi) on the promotion of dendritic development by CD47. Transfection with an expression vector, siRNAfrg-GFP\#2, for an siRNA specific for mouse FRG mRNA (nucleotides 2000-2018) resulted in a marked decrease in the amount of exogenously expressed mouse FRG in transfected HEK293 cells (Fig. 8A). Transfection of hippocampal neurons with the siRNAfrg-GFP\#2 vector prevented the increases in both the number of primary dendrites per neuron and the number of branches from the longest primary dendrite induced by forced expression of CD47, without affecting these parameters in neurons not overexpressing CD47 (Fig. $8 B-D$ ). In contrast, transfection with siRNAfrg-GFP\#2 failed to prevent the formation of dendritic filopodia and spines induced by overexpression of CD47 and exposure to SHPS-1-Fc (Fig. 8E). We also designed another expression vector, siRNAfrg-GFP\#1, for an siRNA that targets a different region of mouse FRG mRNA (nucleotides 1138-1156). Transfection with siRNAfrg-GFP\#1 reduced the amount of exogenous mouse FRG in transfected HEK293 cells and prevented the CD47-induced increases in both the number of primary dendrites per neuron and the number of branches
A

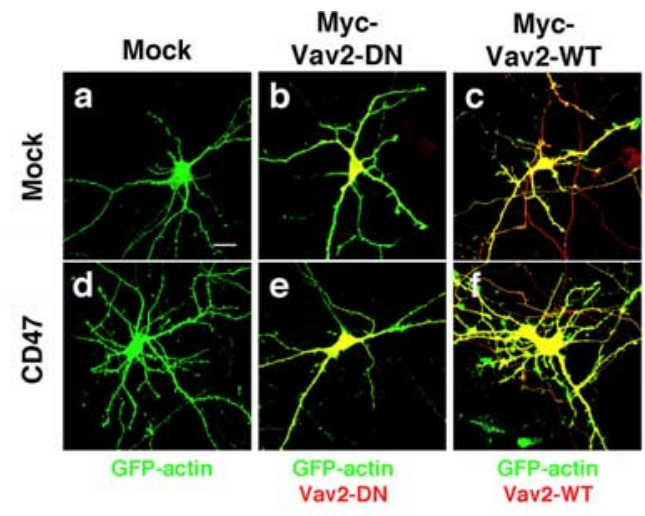

B

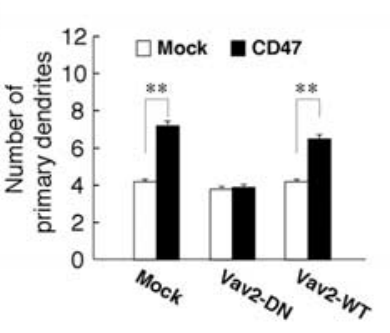

C

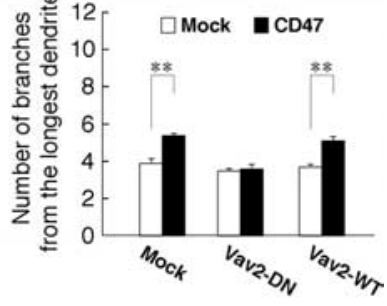

D

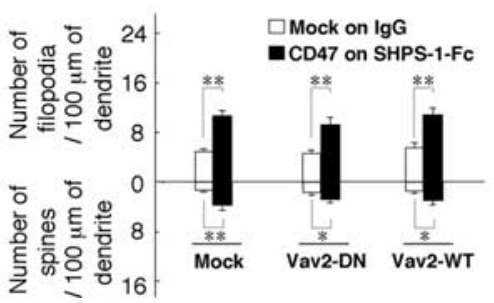

Figure 7. Participation of Vav2 in the promotion of dendritic development by CD47. A, Hippocampal neurons from WT mice were transfected at 4 DIV with a vector for GFP-actin, a vector for CD47 ( $\boldsymbol{d}-\boldsymbol{f})$ or the corresponding empty vector $(\boldsymbol{a}-\boldsymbol{c})$, and a vector for Myc epitopetagged Vav2-DN $(\boldsymbol{b}, \boldsymbol{e})$, a vector for Myc-Vav2-WT $(\boldsymbol{c}, \boldsymbol{f})$, or the corresponding empty vector $(\boldsymbol{a}, \boldsymbol{d})$. At $7 \mathrm{DIV}$, neurons were fixed and stained with an mAb to Myc (red), and GFP-actin fluorescence was monitored (green). Neurons were also stained with an $\mathrm{mAb}$ to CD47 to confirm its expression (data not shown). Scale bar, $20 \mu \mathrm{m} . \boldsymbol{B}, \boldsymbol{C}$, The number of primary dendrites per neuron $(\boldsymbol{B})$ and that of branches from the longest primary dendrite of each neuron $(\boldsymbol{C})$ were determined for neurons treated as in $A$. Data are means $\pm S E$ of values obtained from a total of 30 neurons in three independent experiments. ${ }^{* *} p<0.01$ (Student's $t$ test). $\boldsymbol{D}$, Neurons transfected as in $\boldsymbol{A}$ were cultured on dishes coated with either SHPS-1-Fc (for those transfected with the CD47 vector) or control human IgG (for those transfected with the corresponding empty vector). At $7 \mathrm{DIV}$, they were fixed and stained with an $\mathrm{mAb}$ to $\mathrm{Myc}$, and the densities of dendritic filopodia and spines were determined. Data are means \pm SE of values obtained from a total of 18 neurons in three independent experiments. ${ }^{*} p<0.05$, ${ }^{* *} p<0.01$ (Student's $t$ test).

from the longest primary dendrite in hippocampal neurons (data not shown).

\section{Discussion}

We have shown that CD47 promotes dendritic and axonal development in hippocampal neurons. Loss of CD47 impaired the formation of dendrites and their branching, whereas forced expression of this protein restored and further promoted dendritic development in cultured $C D 47^{-1-}$ neurons. In addition, deficiency of CD47 impaired the elongation of axons and their branching as well as the formation of growth cones. The impairment in dendritic development of $C D 47^{-/-}$hippocampal neurons was evident at an early stage of culture (3-7 DIV) but not at later stages (14-21 DIV). Forced expression of CD47 also increased the number and branching of dendrites in WT neurons at 
A
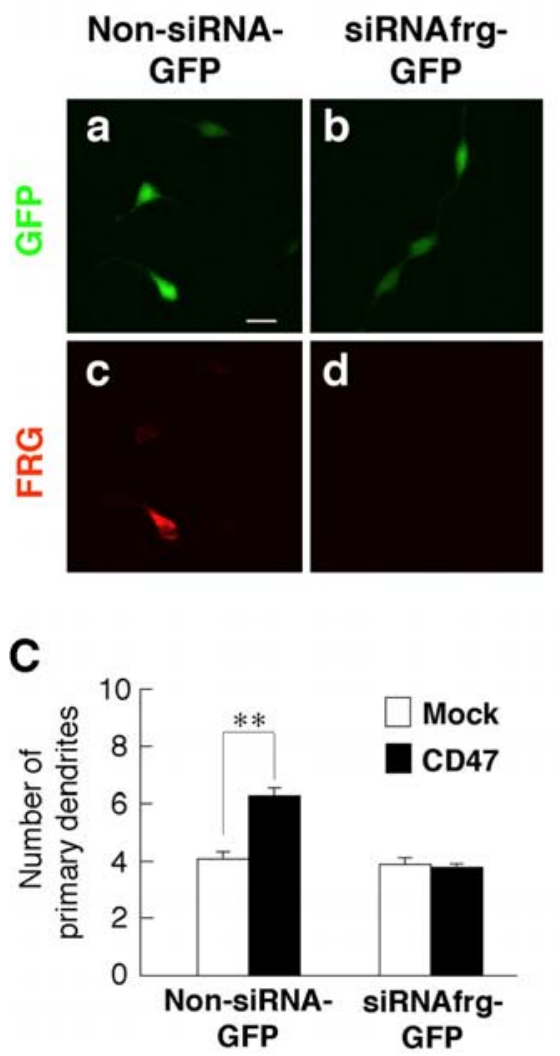

B
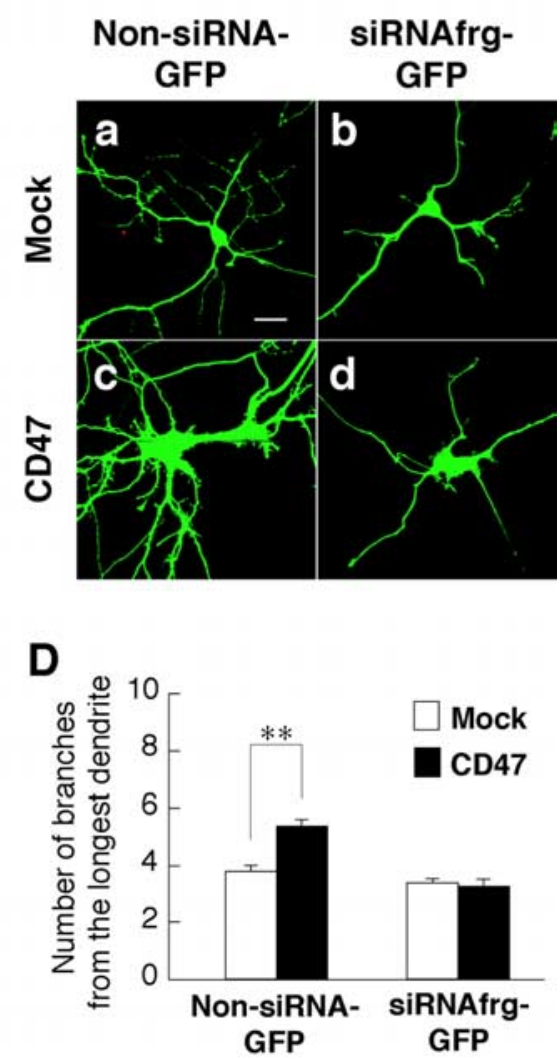

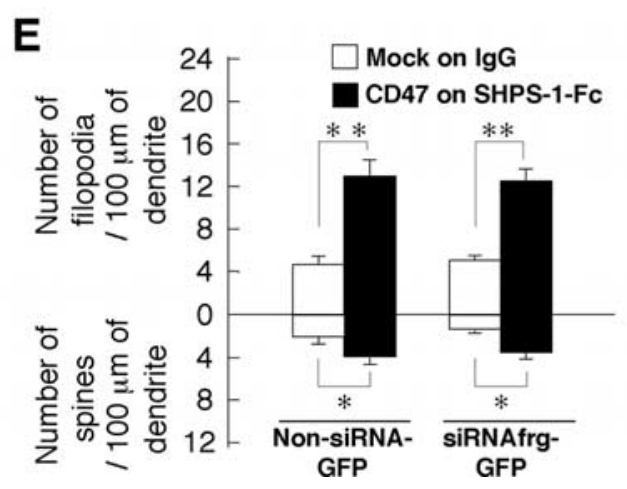

Figure 8. Effects of RNAi-mediated depletion of FRG on CD47-induced dendritic development. $A, H E K 293$ cells were transfected with an expression vector for mouse FRG and either siRNAfrg-GFP\#2 $(\boldsymbol{b}, \boldsymbol{d})$ or the corresponding GFP vector (Non-siRNA-GFP; $\boldsymbol{a}, \boldsymbol{c}$ ). Forty-eight hours after transfection, the cells were fixed and stained with pAbs to FRG $(\boldsymbol{c}, \boldsymbol{d})$ and monitored for GFP fluorescence $(\boldsymbol{a}$, $\boldsymbol{b})$. Scale bar, $20 \mu \mathrm{m}$. $\boldsymbol{B}$, Hippocampal neurons from WT mice were transfected at 4 DIV with siRNAfrg-GFP\#2 $(\boldsymbol{b}, \boldsymbol{d})$ or the corresponding GFP vector $(\boldsymbol{a}, \boldsymbol{c})$, as well as with a vector for $\operatorname{CD} 47(\boldsymbol{c}, \boldsymbol{d})$ or the corresponding empty vector $(\boldsymbol{a}, \boldsymbol{b})$. Neurons were fixed, and the expression of GFP was monitored at 7 DIV. Neurons were also stained with an mAb to CD47 (data not shown). Scale bar, $20 \mu \mathrm{m}$. $\boldsymbol{C}, \boldsymbol{D}$, The number of primary dendrites per neuron $(\boldsymbol{C})$ and that of branches from the longest primary dendrite of each neuron $(\boldsymbol{D})$ were determined for neurons treated as in $\boldsymbol{B}$. Data are means \pm SE of values from a total of $20-30$ neurons in three independent experiments. ${ }^{* *} p<0.01$ (Student's $t$ test). $\boldsymbol{E}$, Hippocampal neurons from WT mice were transfected as in $\boldsymbol{B}$ and cultured on dishes coated with either SHPS-1-Fc (for those transfected with the CD47 vector) or control human lgG (for those transfected with the corresponding empty vector). At 7 DIV, neurons were fixed and stained with an mAb to CD47, and the densities of dendritic filopodia and spines were determined. Data are means $\pm S E$ of values obtained from a total of 18 neurons in three independent experiments. ${ }^{*} p<0.05,{ }^{* *} p<0.01$ (Student's $t$ test).

3-7 DIV but not at 14-21 DIV. These results suggest that CD47 efficiently promotes dendritic and axonal development, in particular, at the early stage of formation of neuronal networks.

We also found that SHPS-1-Fc markedly promoted the formation of dendritic filopodia and spines in CD47-overexpressing hippocampal neurons at 7 DIV but not at 14 or 21 DIV. Given that formation of dendritic filopodia precedes that of dendritic spines (Ziv and Smith, 1996), these results further suggest that CD47, through its interaction with SHPS-1, promotes filopodium formation, resulting in efficient formation of spines and subsequent formation of synapses. We have shown previously that CD47 is localized predominantly to the surface of dendrites in hippocampal neurons, whereas SHPS-1 was detected at the surface of both axons and dendrites (Ohnishi et al., 2005). On the basis of our present results, we suggest that CD47 promotes the formation of filopodia on dendrites through its interaction with SHPS-1 expressed on neighboring axons, thereby contributing to subsequent formation of mature dendritic spines particularly at the early stage of development of neuronal networks. In contrast, because the effect of SHPS-1-Fc on filopodium or spine formation was not apparent at the later stage of culture (14 or $21 \mathrm{DIV})$, the functional role of engagement of SHPS-1 with CD47 after formation and maturation of neuronal networks remains unknown. However, synaptic connections between neurons are thought to be actively disrupted and reconnected even in mature CNS (De Paola et al., 2006; Stettler et al., 2006). Therefore, it is feasible that engagement of SHPS-1 with CD47 participates in the dendrite plasticity of neurons through regulation of formation of filopodia or spines. SHPS-1-Fc failed to increase the number of primary dendrites or that of branches from the longest primary dendrite in either mock-transfected or CD47expressing neurons at 7 DIV (supplemental Fig. 3, available at www.jneurosci.org as supplemental material). These data indicate that engagement by CD47 of SHPS-1 is unlikely to promote dendrite formation and branching.

$C D 47^{-/-}$mice exhibit impaired memory retention and defective long-term potentiation (Chang et al., 1999). Such phenotypes might be attributable to impairment of dendritic and axonal development resulting from the loss of CD47. In contrast to our observations with cultured hippocampal neurons, we did not find marked impairment of dendritic or axonal development in $C D 47^{-1-}$ hippocampus in vivo, whereas the branching of dendrites of hippocampal neurons at mutant mice at $\mathrm{P} 0-\mathrm{P} 1$ was slightly impaired in vivo by staining of fixed hippocampal sections with $1,1^{\prime}$-dioctadecyl-3,3,3',3'tetramethylindocarboyanine perchlorate (data not shown).

We also shown that inhibition of Rac or Cdc42 prevented the effects of forced expression of CD47 on the formation of dendrites and their branching in cultured hippocampal neurons. 
Moreover, we showed that either Rac or Cdc42 in hippocampal neurons are indeed activated by forced expression of CD47 by the use of FRET imaging. We showed previously that forced expression of CD47 also induced a marked increase of the activity of Rac or Cdc42 in COS-7 cells by the use of pull-down assay (Miyashita et al., 2004). Together, our results suggest that Rac as well as Cdc42 participate in the signaling downstream of CD47 in hippocampal neurons. The formation of dendritic filopodia and spines induced by SHPS-1-Fc in CD47-overexpressing neurons was also prevented by inhibition of $\mathrm{Cdc} 42$. These data are also consistent with our previous observations in the neuroblastoma cell line N1E-115 (Miyashita et al., 2004). Rac and Cdc42 are thought to regulate axonal or dendritic development in neurons through their effects on the arrangement of the actin cytoskeleton (Luo et al., 1997; Takai et al., 2001; Govek et al., 2005). However, it has remained unclear which cell surface proteins participate in coupling of extracellular stimuli to activation of these small GTPbinding proteins in such regulation. Our data now suggest that CD47 is such a protein in hippocampal neurons.

Our data have also provided insight into the signaling pathway that links CD47 to Rac or Cdc42 in hippocampal neurons. Inhibition by PP2 or Csk of an Src family kinase prevented the promotion of dendritic development by CD47. In addition, loss of CD47 markedly reduced the activation of c-Src in hippocampal neurons, whereas forced expression of CD47 induced activation of this kinase. These results suggest that c-Src or another Src family kinase participates in CD47-induced dendritic development. The mechanism by which CD47 promotes activation of an Src family kinase is unknown. CD47 interacts with integrins through its extracellular region and participates in integrinmediated biological responses in non-neural cells (Brown and Frazier, 2001). Various integrin subunits, including $\alpha \mathrm{v}, \alpha 2, \alpha 5$, $\beta 1$, and $\beta 3$, are expressed in the hippocampus (Bi et al., 2001; Chan et al., 2003). In addition, integrin engagement induces recruitment and subsequent activation of $\mathrm{c}-\mathrm{Src}$, which in turn phosphorylates downstream signaling molecules (Brunton et al., 2004). CD47 may thus induce activation of c-Src through its interaction with integrins in hippocampal neurons. Unexpectedly, however, inhibition of integrin signaling by echistatin or mAbs to integrin $\beta 1$ or $\beta 3$ subunits failed to prevent the stimulatory effect of CD47 on dendritic development in hippocampal neurons (data not shown). It is thus possible that CD47 induces activation of c-Src in an integrin-independent manner in hippocampal neurons. Indeed, CD47 was shown previously to promote T-cell activation in a manner independent of integrins ( Reinhold et al., 1997).

Inhibition of either Vav2 or FRG prevented the promotion of dendritic development by CD47. Vav2 activates Rac and Cdc42 (Abe et al., 2000; Marignani and Carpenter, 2001), as does FRG (Kubo et al., 2002; Miyamoto et al., 2003; Fukuhara et al., 2004). Our results thus suggest that Vav2 and FRG participate in the stimulatory effect of CD47 on dendritic development in hippocampal neurons. Given that both of these GEFs are activated as a result of tyrosine phosphorylation by c-Src (Marignani and Carpenter, 2001; Miyamoto et al., 2003; Servitja et al., 2003), it is most likely that the activation of c-Src by CD47 results in the tyrosine phosphorylation and activation of Vav2 and FRG in hippocampal neurons. In contrast, it is also possible that Src, Vav2, and FRG may act in parallel. The promotion of filopodium and spine formation by CD47 and SHPS-1-Fc was not prevented by inhibition of Src, Vav2, or FRG, however, suggesting that another as yet unidentified GEF for Cdc42 participates in this effect of CD47 and SHPS-1-Fc. We also tested the effects of a dominant-

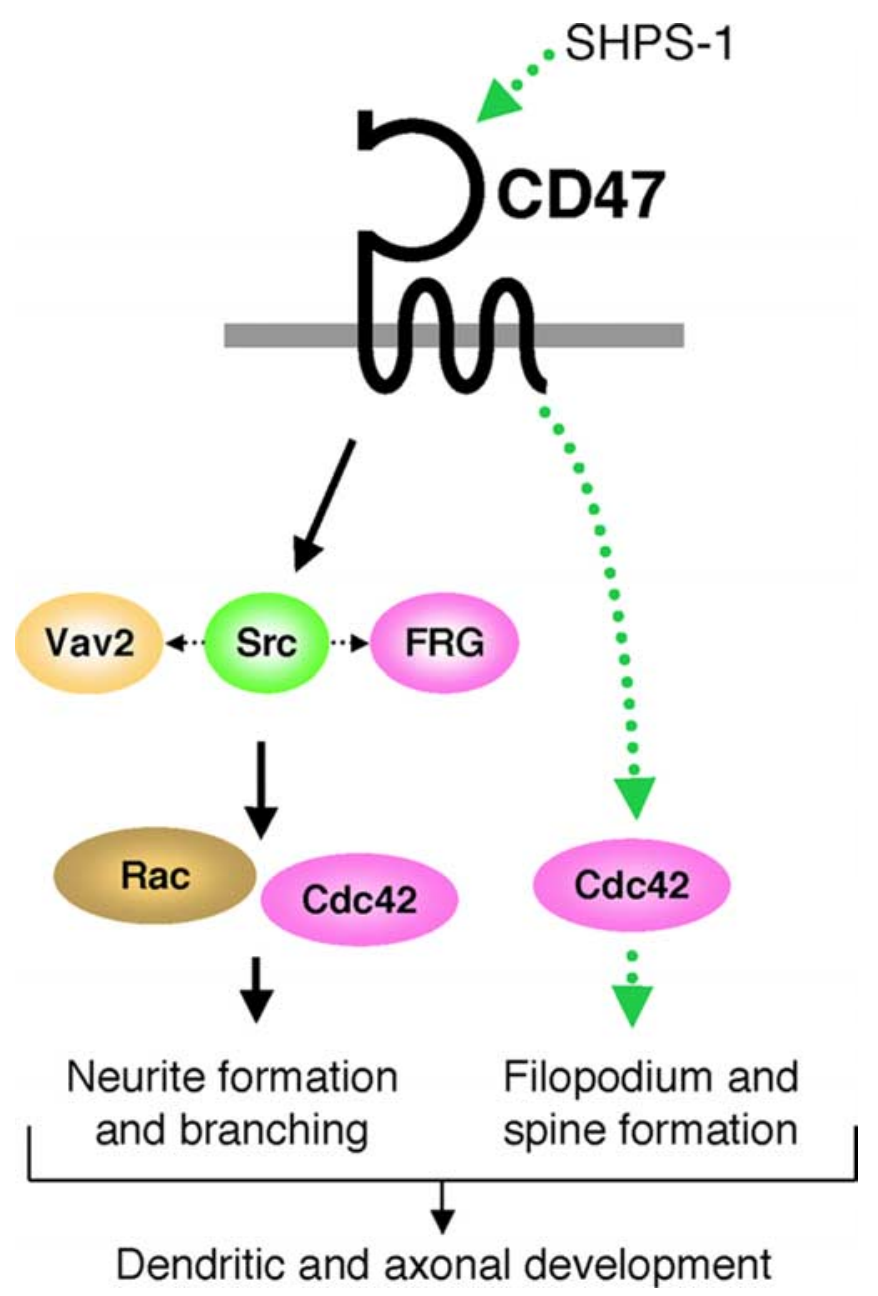

Figure 9. Model for the promotion of neuronal development by CD47. The activation by CD47 of an Src family kinase may result in the tyrosine phosphorylation of Vav2 and FRG, the consequent activation of $\operatorname{Rac}$ and $C \mathrm{dc} 42$, and the promotion of neurite formation and branching. Src, Vav2, and FRG may also act in parallel at the signaling downstream of CD47. Interaction of SHPS-1 with CD47 promotes the formation of dendritic filopodia and spines possibly through a different signaling pathway involving (dc42 (dotted green lines), although the mechanism of such an effect is unknown.

negative mutant of Tiam1 (T-cell lymphoma invasion and metastasis 1), another GEF for Rac (Michiels et al., 1997). However, expression of this mutant failed to prevent the CD47-induced increases in the number of primary dendrites per neuron and branches from the longest primary dendrite (data not shown). It also failed to prevent the formation of dendritic filopodia and spines induced by CD47 and SHPS-1-Fc (data not shown).

In summary, we propose the following model for the mode of CD47 action in the promotion of dendritic and axonal development in hippocampal neurons (Fig. 9). CD47 activates an Src family kinase, such as c-Src, directly or indirectly, which likely results in the tyrosine phosphorylation of Vav2 and FRG and the consequent activation of Rac and Cdc42. Alternatively, Src, Vav2, and FRG may also act in parallel at the signaling downstream of CD47. Interaction of SHPS-1 with CD47 promotes the formation of dendritic filopodia and spines potentially through activation of Cdc42, although the mechanism for such activation is unknown.

\section{References}

Abe K, Rossman KL, Liu B, Ritola KD, Chiang D, Campbell SL, Burridge K, Der CJ (2000) Vav2 is an activator of Cdc42, Rac1, and RhoA. J Biol Chem 275:10141-10149. 
Bi X, Lynch G, Zhou J, Gall CM (2001) Polarized distribution of alpha5 integrin in dendrites of hippocampal and cortical neurons. J Comp Neurol 435:184-193.

Brown EJ, Frazier WA (2001) Integrin-associated protein (CD47) and its ligands. Trends Cell Biol 11:130-135.

Brown MT, Cooper JA (1996) Regulation, substrates and functions of src. Biochim Biophys Acta 1287:121-149.

Brummelkamp TR, Bernards R, Agami R (2002) A system for stable expression of short interfering RNAs in mammalian cells. Science 296:550-553.

Brunton VG, MacPherson IR, Frame MC (2004) Cell adhesion receptors, tyrosine kinases and actin modulators: a complex three-way circuitry. Biochim Biophys Acta 1692:121-144.

Chan CS, Weeber EJ, Kurup S, Sweatt JD, Davis RL (2003) Integrin requirement for hippocampal synaptic plasticity and spatial memory. J Neurosci 23:7107-7116.

Chang HP, Lindberg FP, Wang HL, Huang AM, Lee EH (1999) Impaired memory retention and decreased long-term potentiation in integrinassociated protein-deficient mice. Learn Mem 6:448-457.

De Paola V, Holtmaat A, Knott G, Song S, Wilbrecht L, Caroni P, Svoboda K (2006) Cell type-specific structural plasticity of axonal branches and boutons in the adult neocortex. Neuron 49:861-875.

Dickson BJ (2002) Molecular mechanisms of axon guidance. Science 298:1959-1964

Fujioka Y, Matozaki T, Noguchi T, Iwamatsu A, Yamao T, Takahashi N, Tsuda M, Takada T, Kasuga M (1996) A novel membrane glycoprotein, SHPS-1, that binds the SH2-domain-containing protein tyrosine phosphatase SHP-2 in response to mitogens and cell adhesion. Mol Cell Biol 16:6887-6899.

Fukuhara T, Shimizu K, Kawakatsu T, Fukuyama T, Minami Y, Honda T, Hoshino T, Yamada T, Ogita H, Okada M, Takai Y (2004) Activation of Cdc42 by trans interactions of the cell adhesion molecules nectins through c-Src and Cdc42-GEF FRG. J Cell Biol 166:393-405.

Govek EE, Newey SE, Van Aelst L (2005) The role of the Rho GTPases in neuronal development. Genes Dev 19:1-49.

Hanke JH, Gardner JP, Dow RL, Changelian PS, Brissette WH, Weringer EJ, Pollok BA, Connelly PA (1996) Discovery of a novel, potent, and Src family-selective tyrosine kinase inhibitor. Study of Lck- and FynTdependent $\mathrm{T}$ cell activation. J Biol Chem 271:695-701.

Honda Z, Suzuki T, Hirose N, Aihara M, Shimizu T, Nada S, Okada M, Ra C, Morita Y, Ito K (1997) Roles of C-terminal Src kinase in the initiation and the termination of the high affinity IgE receptor-mediated signaling. J Biol Chem 272:25753-25760.

Huang AM, Wang HL, Tang YP, Lee EH (1998) Expression of integrinassociated protein gene associated with memory formation in rats. J Neurosci 18:4305-4313.

Itoh RE, Kurokawa K, Ohba Y, Yoshizaki H, Mochizuki N, Matsuda M (2002) Activation of Rac and Cdc42 video imaged by fluorescent resonance energy transfer-based single-molecule probes in the membrane of living cells. Mol Cell Biol 22:6582-6591.

Jan YN, Jan LY (2003) The control of dendrite development. Neuron 40:229-242.

Jiang P, Lagenaur CF, Narayanan V (1999) Integrin-associated protein is a ligand for the P84 neural adhesion molecule. J Biol Chem 274:559-562.

Kawakatsu T, Ogita H, Fukuhara T, Fukuyama T, Minami Y, Shimizu K, Takai Y (2005) Vav2 as a Rac-GDP/GTP exchange factor responsible for the nectin-induced, c-Src- and Cdc42-mediated activation of Rac. J Biol Chem 280:4940-4947.

Kodama A, Matozaki T, Fukuhara A, Kikyo M, Ichihashi M, Takai Y (2000) Involvement of an SHP-2-Rho small G protein pathway in hepatocyte growth factor/scatter factor-induced cell scattering. Mol Biol Cell 11:2565-2575.

Kubo T, Yamashita T, Yamaguchi A, Sumimoto H, Hosokawa K, Tohyama M (2002) A novel FERM domain including guanine nucleotide exchange factor is involved in Rac signaling and regulates neurite remodeling. J Neurosci 22:8504-8513.

Lindberg FP, Bullard DC, Caver TE, Gresham HD, Beaudet AL, Brown EJ
(1996) Decreased resistance to bacterial infection and granulocyte defects in IAP-deficient mice. Science 274:795-798.

Luo L, Jan LY, Jan YN (1997) Rho family GTP-binding proteins in growth cone signalling. Curr Opin Neurobiol 7:81-86.

Marignani PA, Carpenter CL (2001) Vav2 is required for cell spreading. J Cell Biol 154:177-186.

Mi ZP, Jiang P, Weng WL, Lindberg FP, Narayanan V, Lagenaur CF (2000) Expression of a synapse-associated membrane protein, P84/SHPS-1, and its ligand, IAP/CD47, in mouse retina. J Comp Neurol 416:335-344.

Michiels F, Stam JC, Hordijk PL, van der Kammen RA, Ruuls-Van Stalle L, Feltkamp CA, Collard JG (1997) Regulated membrane localization of Tiam1, mediated by the NH2-terminal pleckstrin homology domain, is required for Rac-dependent membrane ruffling and C-Jun NH2-terminal kinase activation. J Cell Biol 137:387-398.

Miyamoto Y, Yamauchi J, Itoh H (2003) Src kinase regulates the activation of a novel FGD-1-related Cdc42 guanine nucleotide exchange factor in the signaling pathway from the endothelin A receptor to JNK. J Biol Chem 278:29890-29900.

Miyashita M, Ohnishi H, Okazawa H, Tomonaga H, Hayashi A, Fujimoto TT, Furuya N, Matozaki T (2004) Promotion of neurite and filopodium formation by CD47: roles of integrins, Rac, and Cdc42. Mol Biol Cell 15:3950-3963.

Ohnishi H, Kubota M, Ohtake A, Sato K, Sano S (1996) Activation of protein-tyrosine phosphatase SH-PTP2 by a tyrosine-based activation motif of a novel brain molecule. J Biol Chem 271:25569-25574.

Ohnishi H, Kaneko Y, Okazawa H, Miyashita M, Sato R, Hayashi A, Tada K, Nagata S, Takahashi M, Matozaki T (2005) Differential localization of Src homology 2 domain-containing protein tyrosine phosphatase substrate-1 and CD47 and its molecular mechanisms in cultured hippocampal neurons. J Neurosci 25:2702-2711.

Polleux F, Morrow T, Ghosh A (2000) Semaphorin 3A is a chemoattractant for cortical apical dendrites. Nature 404:567-573.

Reinhold MI, Lindberg FP, Plas D, Reynolds S, Peters MG, Brown EJ (1995) In vivo expression of alternatively spliced forms of integrin-associated protein (CD47). J Cell Sci 108:3419-3425.

Reinhold MI, Lindberg FP, Kersh GJ, Allen PM, Brown EJ (1997) Costimulation of $\mathrm{T}$ cell activation by integrin-associated protein (CD47) is an adhesion-dependent, CD28-independent signaling pathway. J Exp Med 185:1-11.

Servitja JM, Marinissen MJ, Sodhi A, Bustelo XR, Gutkind JS (2003) Rac1 function is required for Src-induced transformation. Evidence of a role for Tiam1 and Vav2 in Rac activation by Src. J Biol Chem 278:34339-34346.

Shamah SM, Lin MZ, Goldberg JL, Estrach S, Sahin M, Hu L, Bazalakova M, Neve RL, Corfas G, Debant A, Greenberg ME (2001) EphA receptors regulate growth cone dynamics through the novel guanine nucleotide exchange factor ephexin. Cell 105:233-244.

Stettler DD, Yamahachi H, Li W, Denk W, Gilbert CD (2006) Axons and synaptic boutons are highly dynamic in adult visual cortex. Neuron 49:877-887.

Takahashi H, Sekino Y, Tanaka S, Mizui T, Kishi S, Shirao T (2003) Drebrin-dependent actin clustering in dendritic filopodia governs synaptic targeting of postsynaptic density-95 and dendritic spine morphogenesis. J Neurosci 23:6586-6595.

Takai Y, Sasaki T, Matozaki T (2001) Small GTP-binding proteins. Physiol Rev 81:153-208.

Tessier-Lavigne M, Goodman CS (1996) The molecular biology of axon guidance. Science 274:1123-1133.

Wahl S, Barth H, Ciossek T, Aktories K, Mueller BK (2000) Ephrin-A5 induces collapse of growth cones by activating Rho and Rho kinase. J Cell Biol 149:263-270.

Whitford KL, Marillat V, Stein E, Goodman CS, Tessier-Lavigne M, Chedotal A, Ghosh A (2002) Regulation of cortical dendrite development by SlitRobo interactions. Neuron 33:47-61.

Ziv NE, Smith SJ (1996) Evidence for a role of dendritic filopodia in synaptogenesis and spine formation. Neuron 17:91-102. 